\title{
The apomorphine test in Parkinsonian syndromes
}

\author{
D F D'Costa, R J Abbott, I F Pye, P A H Millac
}

\begin{abstract}
The dopamine receptor agonist apomorphine has been used successfully to treat on-off swings in Parkinson's disease. Its value as a predictor of dopa responsiveness in idiopathic Parkinson's disease (IPD) was assessed and its potential role in differentiating IPD from the Parkinsonian plus syndromes (PPS) of multisystem atrophy, progressive supranuclear palsy and olivopontocerebellar atrophy was investigated. The response to an injection of apomorphine was observed in 20 patients with IPD and eight with PPS after being off levodopa for 12 hours. Patients were reassessed after taking levodopa for one month. Nineteen of the 20 patients $(95 \%)$ with IPD showed a positive response to apomorphine and $18(90 \%)$ to oral levodopa. In the PPS group, two patients $(25 \%)$ responded to the apomorphine injection but not to oral levodopa. Apomorphine produced severe drowsiness in the PPS patients. It is suggested that the test can predict dopa responsiveness in IPD and may be of help in confirming a doubtful diagnosis. It has potential value in differentiating IPD from PPS.
\end{abstract}

Apomorphine, a powerful dopamine receptor agonist, is a useful treatment for the on-off fluctuations in Parkinson's disease. ${ }^{12}$ Recent reports $^{3-5}$ suggest that it might be used to predict levodopa responsiveness in Parkinsonian syndromes. Our study was set up to investigate this possible application further and to see whether idiopathic Lewy body Parkinson's disease (IPD) could be distinguished from the Parkinsonian plus syndromes (PPS) of multisystem atrophy (MSA), progressive supranuclear palsy (PSP) and olivopontocerebellar atrophy (OPCA). In addition we wanted to assess whether an uncertain diagnosis of IPD could be confirmed.

Department of Neurology, Leicester Royal Infirmary, Leicester, UK D F D'Costa R J Abbott I F Pye

P A H Millac

Correspondence to: Dr D'Costa, Leicester General Hospital, Leicester LE5 4QT, UK.

Received 15 April 1990 and in final revised form 9 January 1991 .

Accepted 25 January 1991

\section{Patients and methods}

We assessed 28 patients - 20 with IPD and eight with PPS-on the basis of a clinical diagnosis and CT brain scans. The diagnosis of IPD was made on classic rest tremor, with bradykinesia and/or rigidity and a response to levodopa treatment (in patients already being treated). The IPD patients had negative CT scans. The diagnosis of PPS was based on the absence of rest tremor, early falls or dementia, abnormalities of eye movements, cerebellar ataxia or pyramidal signs. This group were also unable to tolerate levodopa and had CT scans showing atrophy on one or more areas. Five patients with IPD were newly diagnosed and had not received levodopa. The PPS group consisted of four patients with MSA, three with PSP and one with OPCA. Patients with impaired renal function or severe dementia were excluded. Informed consent was obtained.

Patients received domperidone (a peripheral dopamine receptor antagonist) $20 \mathrm{mg}$ eight hourly, 24 hours before the test, and throughout its duration to prevent side effects. All anti-Parkinsonian medication (levodopa, anticholinergics, bromocriptine, selegiline) was discontinued 12 hours before the test. Motor function was assessed by the tap test and the walking test. In the tap test, the patient had to tap for 15 seconds between two plates $20 \mathrm{~cm}$ apart with a metal stylus connected to an electronic counter ${ }^{6}$ using the most affected hand. The walking test consisted of the time taken to rise from an armless chair, walk six metres, turn and return. A mean of three scores was recorded for each test. Assessments were made as a baseline and then every 15 minutes after a subcutaneous injection of $1 \mathrm{mg}$ of apomorphine. The dose was increased sequentially to $2,4,5,8$ or $10 \mathrm{mg}$ until either a positive response occurred or intolerance developed, the minimum time between each injection being 90 minutes. A positive response was defined as a change of $>15 \%$ from baseline in either tapping or walking. Patients were then given oral levodopa the doses of which varied according to clinical severity. All patients were assessed at the end of one month whilst on levodopa. The tap test (a mean of 3 scores) and walking test (mean of 3 scores) were performed one hour after the morning dose of levodopa. Sequential recordings were not made. Age and sex matched controls with no neurological disability were also assessed to try and define normal tap and walking scores.

Statistical methods: non-parametric methods using the Wilcoxon signed rank test and Spearman's rank correlation were used to analyse the results. The significance value of the correlations was calculated using a $t$ test.
Results

The results of the two groups are shown in the groups was 50 and 66 years and the mean duration of the disease 3.8 and 3.0 years respectively. Nineteen patients (95\%) with IPD and two patients (25\%) with PPS showed a positive response to apomorphine.

In the IPD group, the baseline tap scores, mean (SEM), (fig 1) of $31 \cdot 3(2 \cdot 2)$ improved by table. The mean age for the IPD and PPS 
Table Characteristics of the two groups

\begin{tabular}{lll}
\hline & IPD & $P P S$ \\
\hline Total number & 20 & 8 \\
Mean age (range) years & $50(35-78)$ & $66(51-77)$ \\
Sex ratio (M/F) & $12 / 8$ & $4 / 4$ \\
$\begin{array}{l}\text { Average duration of disease- } \\
\text { in years }\end{array}$ & $3 \cdot 8(1-16)$ & $3(1-8)$ \\
$\begin{array}{l}\text { Mean apomorphine dose (mg) } \\
\text { Positive responders to }\end{array}$ & $3 \cdot 7(1-10)$ & $3 \cdot 3(1-8)$ \\
$\quad$ apomorphine (total) & 19 & 2 \\
$\begin{array}{l}\text { Positive responders to } \\
\text { levodopa (total) }\end{array}$ & 18 & $\star 2$ \\
\hline
\end{tabular}

IPD = Idiopathic Parkinson's disease, PPS = Parkinsonian plus syndromes. ${ }^{\star}$ These two patients had a negative response plus syndromes.
to apomorphine.

$27 \cdot 6 \%$ (range $15-75 \%$ ) to 40.0 (3.0) after apomorphine and to $38.3(3.0)$ on levodopa. The walking times (fig 2) were $31.8(6.6)$ seconds (baseline), 21.0 (4.0) seconds after apomorphine, and $22 \cdot 1(5 \cdot 1)$ seconds on levodopa. The improvement in the mean tap score and walking times after apomorphine and levodopa was highly significant $(p<0.01)$. The mean (SEM) scores for age and sex matched controls with no neurological disability were $57 \cdot 7(1 \cdot 7)$ for tapping and $13.5(0.5)$ for walking. Thus even after treatment, the scores were below that of controls. The mean apomorphine dose required to elicit an optimal response was $3.7 \mathrm{mg}$. Three patients required $8 \mathrm{mg}$ and one required $10 \mathrm{mg}$ to show a response. This response was noted within 15 minutes of the injection in all but three of the responders in whom the onset took place at 30 minutes. The duration of response was 75 minutes (range 45-120). Eighteen of the 19 responders to apomorphine showed a similar positive response to levodopa. There was a

Figure 1 Mean Tap Scores $( \pm \mathrm{sem})$ at baseline off treatment $(A)$, after apomorphine $(B)$ and after levodopa $(C)$ in the IPD and PPS groups. $D=$ normal controls. $I P D=$ Idiopathic Parkinson's Disease. $P P S=$ Parkinson's plus syndromes.

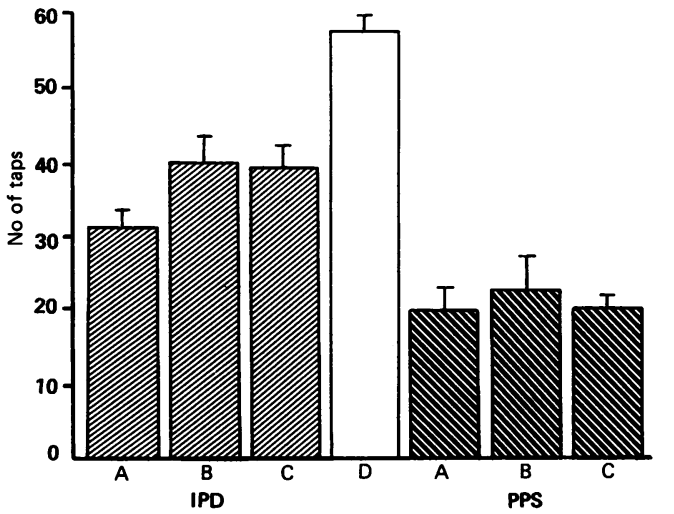

Figure 2 Mean Walking Scores ( \pm sem) at baseline off treatment $(A)$, after apomorphine $(B)$ and after levodopa $(C)$ in the IPD and PPS groups. $D=$ normal controls. $I P D=$ Idiopathic Parkinson's Disease. PPS = Parkinson's plus syndromes. highly significant $(\mathrm{p}<0.001)$ positive correlation between the changes in tapping after apomorphine and levodopa $(r=0.88)$, and between the changes in walking after apomorphine and levodopa $(r=0.96)$. There was a negative correlation between tapping scores and walking times after apomorphine $(\mathrm{r}=-0.64)(\mathrm{p}<0.005)$ and after levodopa $(\mathrm{r}=-0.49)(\mathrm{p}<0.05)$ reflecting that as tapping scores improved, walking times were reduced. Two responders to the tap test showed no change in walking. One patient showed a positive response only in walking and not tapping - as the lower limbs were mainly involved. Only one patient $(5 \%)$ failed to respond to either apomorphine or levodopa. A few patients noted a significant improvement in rest tremor after apomorphine.

In the PPS group, two out of eight $(25 \%)$ responded to apomorphine but neither of these responded to oral levodopa. The mean duration of benefit ( 75 minutes) was similar to the IPD group. The responses to apomorphine were quite dramatic -one patient with MSA for four years showed a $50 \%$ improvement in tapping and $30 \%$ improvement in walking. The other patient with OPCA for two years showed a $20 \%$ improvement in both tapping and walking. Two other patients who obtained no benefit with apomorphine improved with levodopa. Of these, one with PSP for eight years showed a $40 \%$ improvement in both tapping and walking with levodopa and the other with MSA for three years a $30 \%$ improvement in walking. The remaining four patients $(50 \%)$ were unable to tolerate oral levodopa preparations developing nausea, vomiting and malaise even with low doses. Baseline tapping, mean (SEM), $20(2 \cdot 8)$, and walking, $22.8(4 \cdot 7)$, scores did not change significantly after apomorphine $22 \cdot 6(4 \cdot 6)$, $20.5(4.5)$ or after levodopa, $20(1 \cdot 7)$, $18 \cdot 1(2 \cdot 2)$.

Adverse reactions to apomorphine were mild in the IPD group and included nausea, vomiting, dizziness, flushing, sweating and pallor. This affected 10 of 20 patients. One patient developed transient priapism. In the PPS group the side effects were more pronounced and occurred in all patients and at lower dosage than for IPD. In addition the PPS group had quite marked drowsiness which was present in all patients.

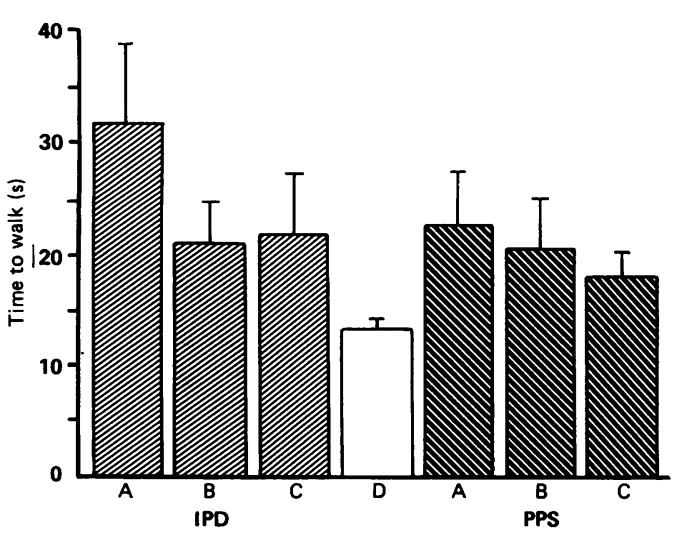

\section{Discussion}

Nineteen patients (95\%) with IPD showed a positive response to apomorphine. Eighteen of these $(94.7 \%)$ showed a similar positive response to prolonged levodopa. The nineteenth patient had a subjective response to apomorphine which could not be confirmed objectively. The test therefore reliably predicts dopa responsiveness in $90 \%$ of patients.

Five of the 20 patients $(25 \%)$ had very early features of IPD and the positive response to apomorphine and levodopa in these patients helped confirm the diagnosis. False negatives, however, may occur particularly in de novo patients. ${ }^{3}$ Five others had previously shown a 
doubtful response to levodopa or were unable to tolerate the preparation. By demonstrating a response to apomorphine these patients then had the opportunity of increasing their current doses or receiving a different levodopa preparation.

The single non-responder to apomorphine and levodopa in the IPD group has shown no response to levodopa infusions and has been unable to tolerate oral levodopa. He was aged 50 and had typical features of IPD with a rest tremor, rigidity and a bradykinetic stooped gait. He had two negative CT scans three years apart. It will be interesting to see whether in the long term he develops PPS. The other dopa non-responding patient was aged 72 . He had classic features of IPD four years ago and he initially responded to treatment, but has not done so recently. He had a $35 \%$ improvement in tapping after apomorphine but no change with levodopa. He is thus a late non-responder and would be suitable for apomorphine.

The mean tap score after apomorphine or levodopa remained $68 \%$ of that of age and sex matched controls. Likewise, the walking time was about $60 \%$ longer than that of controls. Despite apparently optimal treatment, a return to normal function was still not achieved.

In the PPS group, two (25\%) showed a positive response to apomorphine but not to oral levodopa whilst two non-responders to apomorphine showed a positive response to levodopa. Thus the test may not be able to predict reliably dopa responsiveness in PPS patients or differentiate IPD from PPS in all cases. Drowsiness appears to be a feature unique to the PPS group even with low doses, and this in itself may help point to a diagnosis of PPS rather than IPD. Four $(50 \%)$ felt a lot worse after the apomorphine with an objective deterioration in their performance. Three of these were unable to tolerate levodopa but one showed an improvement in walking with levodopa.

In the study by Hughes et $a l l^{3}$ apomorphine accurately predicted the response to long-term levodopa in $90 \%$ of the Parkinsonian patients. We obtained a similar result in our IPD patients. There will thus still be $10 \%$ of patients with a negative response who will respond to prolonged levodopa-hence a negative response should not rule out a trial of levodopa.

In the PPS group, our findings differed from those of Hughes et al. They obtained two falsenegatives out of 35 patients and no falsepositives. We observed two false-negatives out of eight patients and two false-positives. Additionally, our PPS patients had a higher incidence of more pronounced side effects than the IPD group unlike the study by Hughes $e t$ al where the two groups had broadly similar side effects. Although we studied only eight patients with PPS, we believe the profound drowsiness after apomorphine helps point to a diagnosis of PPS.

Hughes et al compared the responses to apomorphine, an oral levodopa challenge and the response to continued levodopa therapy. They found a similar sensitivity between apomorphine and a single levodopa challenge. We did not use the single levodopa challenge. Our study compared responses to apomorphine and prolonged levodopa and we used a maximum of $10 \mathrm{mg}$ of apomorphine as did others ${ }^{7}$ compared with $4.5 \mathrm{mg}$ by Hughes $e t$ al. The low doses used by Hughes et al might explain some of the false-negatives they obtained. Our results therefore confirm their findings that the apomorphine test can be used as a quick and reliable test to predict dopa responsiveness in IPD. In addition it may help when the diagnosis of IPD is in doubt or if patients do not respond to levodopa. The drowsiness produced by low doses of apomorphine may point towards a diagnosis of PPS rather than IPD. A positive test would enable early institution of Selegeline which may delay the need for levodopa. ${ }^{8}$ A negative test may also help avoid prolonged trials with oral levodopa which may give rise to adverse reactions and no clear cut response. The test is easy to perform and can be completed in a short period of time and has been likened to the edrophonium test in myasthenia. ${ }^{4}$ We expect the use of the test to become more widespread.

1 Stibe CMH, Lees AJ, Kempster PA, Stern GM. Subcutaneous apomorphine in parkinsonian on-off oscillations. Lancet 1988;i:403-6.

2 Chaudhuri KR, Critchley P, Abbott RJ, Pye IF, Millac PAH. Subcutaneous apomorphine for on-off oscillations in parkinson's disease. Lancet 1988;i:1260.

3 Hughes AJ, Lees AJ, Stern GM. Apomorphine test to predict dopaminergic responsiveness in parkinsonian syndromes. Lancet 1990;336:32-4.

4 Barker R, Duncan J, Lees AJ. Subcutaneous apomorphine as a diagnostic test for dopaminergic responsiveness in parkinsonian syndromes. Lancet 1989;i:675.

5 Oertel WH, Gasser T, Ippisch R, Trenkwalder C, Poewe W. Apomorphine test for dopaminergic responsiveness. Lancet 1989;i:1263.

6 Wyke $M$. Influence of direction on the rapidity of bilatera arm movements. Neuropsychologia 1969;7:189-94.

7 Rascol O, Senard JM, Rascol A, Montastruc JL. Apomorphine test in parkinsonian syndromes. Lancet 1990, 336:518.

8 The Parkinson study group. Effect of deprenyl on the progression of disability in early Parkinson's disease. $N$ Engl J Med 1989;321:1364-71. 